\title{
How Augmented Reality Affects Advertising Effectiveness: An Abstract
}

\author{
Shuai Yang, Sixing Chen, and Jeffrey R. Carlson
}

\begin{abstract}
Despite marketing practitioners' increasing application of augmented reality (AR) to communication and brand-image development, research related to how AR influences consumers' advertising perceptions has remained underdeveloped. The primary purpose of this research is to explore how and why augmented reality influences advertising effectiveness.

A field experiment (Study 1) provides evidence that AR advertisements positively influence consumers' purchase intention and that this effect is mediated by attitude toward the ad and the brand. In addition, an eye-tracking experiment (Study 2) demonstrates that AR advertisements positively affect consumers' attitude toward the ad and that this effect is mediated by an increase in their curiosity and visual attention toward the ad. By comparing traditional advertising to AR advertising, this research has practical and important implications for advertisers. Further, the use of eye tracking provides managerial implications for advertisers in addition to providing an example of how to measure AR advertising effectiveness.
\end{abstract}

\footnotetext{
S. Yang

Donghua University, Shanghai, China

e-mail: shuai.yang@dhu.edu.cn

S. Chen

Hunan University, Changsha, China

e-mail: sixingchen@hnu.edu.cn

J. R. Carlson $(\square)$

University of Richmond, Richmond, VA, USA

e-mail: jcarlso2@richmond.edu
} 